\title{
Analysis of functionally graded plates subjected to hygro-thermo-mechanical loads
}

\author{
Wu Zhen ${ }^{1}$, Ma Yuting ${ }^{1}$, Ren Xiaohui ${ }^{1}$, S. H. Lo ${ }^{2 *}$ \\ ${ }^{1}$ Key Laboratory of Liaoning Province for Composite Structural Analysis of Aerocraft and \\ Simulation, Shenyang Aerospace University, Shenyang 110136, China \\ ${ }^{2}$ Department of Civil Engineering, University of Hong Kong, Pokfulam Road, Hong Kong, China
}

\begin{abstract}
Analytical solution taking into account the transverse normal strain is developed for the analysis of the mechanical and hygrothermal behaviors of functionally graded material (FGM) plates. In order to include the transverse normal deformation, the hygrothermal deformation through the thickness due to thermal and moisture loads is introduced in the transverse displacement field. Employing the bounding surface free traction condition, effects of transverse normal hygrothermal deformation can be incorporated in the in-plane displacement field. Moreover, there are only five displacement variables in the proposed model as transverse normal deformations due to hygrothermal loads are absorbed in the generalized force vector. By analyzing the hygrothermal behaviors of FGM plates, the accuracy and efficiency of the proposed model are assessed. Moreover, the effects of temperature, moisture concentration, transverse normal strain, volume fraction distributions and side-to-thickness ratios on the displacements and the stresses of FGM plates have all been studied.
\end{abstract}

Keywords: Analytical model; functionally graded plate; transverse normal strain; hygro-thermo-mechanical loads.

\footnotetext{
${ }^{*}$ Corresponding author. Tel.: +852 28591977.

† E-mail address: hreclsh@, hku.hk (S.H. Lo)
} 


\section{Introduction}

The functionally graded materials (FGMs) is generally composed of metal and ceramic in which metal provides the toughness and strength at low temperature while ceramic can offer a high resistance to heat at elevated temperature. Most FGM structures are subjected to changing environmental conditions during both initial fabrication and service life. As a result, internal stresses in FGM structures are generated with the change of hygrothermal environment, which will induce large deformation and even cause failure of the structures. Displacements and stresses in the functionally graded material structures subjected to mechanical and hygrothermal loads have been widely studied in the past, which are still the subject of research interest in recent years [1-3].

Based on the first-order shear deformation theory and von Karman's assumptions, Sundararajan et al. [4] studied the free vibration of FGM plates subjected to thermal load. In terms of the first-order theory, Nguyen-Xuan et al. [5] proposed an edge-based smoothed finite element method for static, free vibration and buckling analysis of FGM plates, in which thermal and mechanical properties of FGM plates are assumed to vary through the thickness by a power rule of the volume fraction of the constituents. Based on the first-order theory, Singha et al. [6] proposed a four-node quadrilateral plate bending element for the analysis of nonlinear behaviors of functionally graded plates subjected to transverse distributed loads. Nguyen-Xuan et al. [7] developed an improved finite element approach based on the first-order theory to study the mechanical/thermal buckling of FGM plates. Transverse shear strains in the first-order shear deformation theory are assumed to be constant through the thickness direction. Thus, the correction factors have to be employed to adjust the transverse shear stiffness. It is noted that the accuracy of results obtained from the first-order theory is strongly influenced by the shear correction factors $[8,9]$. In order to represent the smooth distribution of transverse shear stresses across the thickness, the higher-order shear deformation models have been developed for the thermomechanical analysis of the FGM plates. 
In order to study the effects of volume fractions and modulus ratio of the constituents on displacements and transverse shear stresses of FGM plates, Reddy [10] presented a theoretical formulation and a finite element model based on a third-order shear deformation [11]. Analytical solution in terms of the third-order model [11] allows parabolic variation of transverse shear strain along the plate thickness and satisfies the transverse shear free conditions on the plate surfaces. Zenkour [12] employed a sinusoidal shear deformation theory (SPT) [13] to study the static behaviors of a simply-supported functionally graded plates subjected to a transverse uniform loads. The sinusoidal shear deformation theory satisfies the traction-free boundary conditions and transverse shear correction factors are not required. On the other hand, Zenkour and Alghamdi [14] extended the model SPT to study the thermoelastic bending behaviors of functionally graded sandwich plates. Employing the sinusoidal shear deformation plate theory, Zenkour [15] investigated the thermoelastic bending behaviors of a simply-supported FGM plate subjected to thermomechanical loading and resting on elastic foundation. Moreover, effects of side-to-thickness ratio, volume fraction distributions and elastic foundation parameters are studied in detail. Subsequently, the model SPT was extended to investigate hygrothermal bending of functionally graded material plates resting on elastic foundations [16]. Applying the sinusoidal shear deformation plate theory in conjunction with the first-order, the third-order, and the classical plate bending theories, Zenkour and Alghamdi [17,18] studied the flexural behaviors of functionally graded sandwich plates subjected to mechanical and thermal loads. Based on the sinusoidal shear deformation plate models, Zenkour et al. [19] studied the bending behaviors of a simply-supported functionally graded viscoelastic sandwich beam resting on Pasternak's elastic foundation. Zenkour and Sobhy [20] further employed the sinusoidal shear deformation plate model and the third-order model to evaluate the static response of simply-supported FGM viscoelastic sandwich plates under transverse uniform loads. Employing the sinusoidal shear deformation plate theory, Zenkour and Sobhy [21] computed the deflection and stresses in functionally graded plates resting on two-parameter elastic foundations. based on higher-order theories 
with five displacement variables, Sobhy [22] investigated the vibration and buckling behaviors of exponentially graded material sandwich plates resting on elastic foundations. By means of the generalized shear deformation models including the third-order model [11], the sinusoidal shear deformation plate model [12], the fifth-order shear deformation model (FiSDT) [23], trigonometric shear deformation model [24], exponential shear deformation model (ESDT) [25], Thai et al. [26] studied the static, dynamic and buckling behaviors of functionally graded material sandwich plates.

It is seen from the literature that the generalized shear deformation models with five displacement parameters are widely used to study the hygrothermal behaviors of the FGM structures [10-26]. However, the transverse normal strain has been neglected in these models. For thermomechanical problems even in the moderate thick plate configuration, transverse normal deformation effect ought to be considered as the effect of out-of-plane thermal deformation is equally important in comparison with those of the in-plane thermal deformation [27-29]. Due to the omission of transverse normal strain, the third-order model with five displacement variables failed to produce accurate results for thermal expansion problems [30]. To extend the generalized higher-order model for hygrothermal analysis of FGM plate, a refined model including transverse normal strain will be developed. Although transverse normal strain is taken into account, there are only five displacement parameters in the proposed model as transverse normal deformations due to hygrothermal loads can be absorbed in the generalized force vector. In the proposed model, the equations of equilibrium are established by means of the principle of minimum total potential energy. To maintain the simplicity of the analytical solution with the same number of displacement parameters, the main idea is to extend the Reddy's solution to simply-supported FGM plates subjected to hygro-thermo-mechanical loads by applying the Navier's technique [31]. Several numerical examples are presented to demonstrate the reliability and accuracy of the proposed model in comparison with other existing higher-order models in the literature. 


\section{Theoretical formulation}

A rectangular FGM plate with length $a$, width $b$ and thickness $h$ is considered. The FGM plate is subjected to transverse loading $q(x, y, \pm h / 2)$ on the surfaces, a temperature field $T(x, y, z)$ and a moisture concentration $C(x, y, z)$. The material properties $\mathrm{M}$ of the FGM plate, such as Young's modulus E, thermal expansion coefficient $\alpha$, and moisture expansion coefficient $\beta$ are given by [12]

$$
\mathrm{M}(z)=\mathrm{M}_{\text {metal }}+\left(\mathrm{M}_{\text {ceramic }}-\mathrm{M}_{\text {metal }}\right)\left(\frac{z}{h}+0.5\right)^{k}
$$

where $\mathrm{M}_{\text {metal }}$ and $\mathrm{M}_{\text {ceramic }}$ represent respectively the corresponding material properties of the metal and the ceramic, and $k$ denotes the volume fraction exponent. The mixture of metal and ceramic with continuously varying volume fraction can avoid the interface problems of laminated composites.

\subsection{Reddy-type higher-order plate theory considering transverse normal hygrothermal strain for the FGM plate (RHSDTC)}

In order to model accurately the hygrothermal behaviors of the FGM plate, a Reddy-type higher order shear deformation plate model taking into account the transverse normal strain (RHSDTC) is proposed in the present work. To include transverse normal effect which is significant for the hygrothermal problems, transverse deformation due to hygrothermal loading is introduced in the out-of-plane displacement field. Thus, the initial displacement field is given by

$$
\begin{aligned}
& u=u_{0}+z u_{1}+z^{2} u_{2}+z^{3} u_{3} \\
& v=v_{0}+z v_{1}+z^{2} v_{2}+z^{3} v_{3} \\
& w=w_{0}+w_{T}+w_{C}
\end{aligned}
$$

where $w_{T}$ is the transverse normal deformation due to thermal load and $w_{C}$ is the transverse normal deformation caused by moisture concentration load, which are given by

$$
\begin{aligned}
& w_{T}(x, y, z)=\alpha_{z} \int T(x, y, z) d z \\
& w_{C}(x, y, z)=\beta_{z} \int C(x, y, z) d z
\end{aligned}
$$


where $\alpha_{z}$ and $\beta_{z}$ are respectively the transverse normal thermal expansion coefficient and the transverse normal moisture expansion coefficient through the thickness of the plate.

The temperature field $T(x, y, z)$ and the moisture concentration $C(x, y, z)$ are given by

$$
\begin{aligned}
& T(x, y, z)=f_{T}(z) \bar{T}(x, y) \\
& C(x, y, z)=f_{C}(z) \bar{C}(x, y)
\end{aligned}
$$

Employing equations (3) and (4), transverse normal deformation due to hygrothermal load can be written as

$$
\begin{aligned}
& w_{T}(x, y, z)=\mathrm{F}_{T}(z) \bar{T}(x, y) \\
& w_{C}(x, y, z)=\mathrm{F}_{C}(z) \bar{C}(x, y)
\end{aligned}
$$

where

$$
\begin{aligned}
& \mathrm{F}_{T}(z)=\alpha_{z} \int f_{T}(z) d z \\
& \mathrm{~F}_{C}(z)=\beta_{z} \int f_{C}(z) d z
\end{aligned}
$$

The transverse shear stresses related linearly with the shear strains are given by

$$
\begin{aligned}
& \tau_{x z}=D_{44}(z) \gamma_{x z} \\
& \tau_{y z}=D_{55}(z) \gamma_{x z}
\end{aligned}
$$

where, $D_{44}(z)=D_{55}(z)=\frac{E(z)}{2(1+v)}, E(z)$ and $v$ are Young's modulus and Poisson's ratio of the FGM plate, respectively. From equation (2), we have

$$
\begin{aligned}
& \gamma_{x z}=u_{1}+2 z u_{2}+3 z^{2} u_{3}+\frac{\partial w_{0}}{\partial x}+\frac{\partial w_{T}}{\partial x}+\frac{\partial w_{C}}{\partial x} \\
& \gamma_{y z}=v_{1}+2 z v_{2}+3 z^{2} v_{3}+\frac{\partial w_{0}}{\partial y}+\frac{\partial w_{T}}{\partial y}+\frac{\partial w_{C}}{\partial y}
\end{aligned}
$$

Employing the top and the bottom surface transverse shear free condition, we have

$$
\begin{aligned}
& \left.\gamma_{x z}\right|_{z= \pm h / 2}=u_{1}+2 z u_{2}+3 z^{2} u_{3}+\frac{\partial w_{0}}{\partial x}+\frac{\partial w_{T}}{\partial x}+\frac{\partial w_{C}}{\partial x}=0 \\
& \left.\gamma_{y z}\right|_{z= \pm h / 2}=v_{1}+2 z v_{2}+3 z^{2} v_{3}+\frac{\partial w_{0}}{\partial y}+\frac{\partial w_{T}}{\partial y}+\frac{\partial w_{C}}{\partial y}=0
\end{aligned}
$$

Applying the zero shear stress boundary conditions at the top and the bottom surfaces, a Reddy-type higher-order plate theory including transverse normal 
hygrothermal strain (RHSDTC) is given by

$$
\begin{aligned}
& u=u_{0}+\Phi_{1} u_{1}+\Phi_{2} \frac{\partial w_{0}}{\partial x}+\Phi_{3} \frac{\partial \bar{T}}{\partial x}+\Phi_{4} \frac{\partial \bar{C}}{\partial x} \\
& v=v_{0}+\Psi_{1} v_{1}+\Psi_{2} \frac{\partial w_{0}}{\partial y}+\Psi_{3} \frac{\partial \bar{T}}{\partial y}+\Psi_{4} \frac{\partial \bar{C}}{\partial x} y \\
& w=w_{0}+w_{T}+w_{C}
\end{aligned}
$$

where

$$
\begin{aligned}
& \Phi_{1}=z-\frac{4 z^{3}}{3 h^{2}}, \Phi_{2}=-\frac{4 z^{3}}{3 h^{2}}, \\
& \Phi_{3}=\frac{z^{2}}{h}\left(\frac{\mathrm{F}_{T}\left(z_{1}\right)-\mathrm{F}_{T}\left(z_{2}\right)}{2}\right)-\frac{4 z^{3}}{3 h^{2}}\left(\frac{\mathrm{F}_{T}\left(z_{1}\right)+\mathrm{F}_{T}\left(z_{2}\right)}{2}\right), \\
& \Phi_{4}=\frac{z^{2}}{h}\left(\frac{\mathrm{F}_{C}\left(z_{1}\right)-\mathrm{F}_{C}\left(z_{2}\right)}{2}\right)-\frac{4 z^{3}}{3 h^{2}}\left(\frac{\mathrm{F}_{C}\left(z_{1}\right)+\mathrm{F}_{C}\left(z_{2}\right)}{2}\right) ; \\
& \Psi_{1}=z-\frac{4 z^{3}}{3 h^{2}}, \Psi_{2}=-\frac{4 z^{3}}{3 h^{2}}, \\
& \Psi_{3}=\frac{z^{2}}{h}\left(\frac{\mathrm{F}_{T}\left(z_{1}\right)-\mathrm{F}_{T}\left(z_{2}\right)}{2}\right)-\frac{4 z^{3}}{3 h^{2}}\left(\frac{\mathrm{F}_{T}\left(z_{1}\right)+\mathrm{F}_{T}\left(z_{2}\right)}{2}\right), \\
& \Psi_{4}=\frac{z^{2}}{h}\left(\frac{\mathrm{F}_{C}\left(z_{1}\right)-\mathrm{F}_{C}\left(z_{2}\right)}{2}\right)-\frac{4 z^{3}}{3 h^{2}}\left(\frac{\mathrm{F}_{C}\left(z_{1}\right)+\mathrm{F}_{C}\left(z_{2}\right)}{2}\right) .
\end{aligned}
$$

where $z_{1}$ and $z_{2}$ are respectively the $z$-coordinate at the bottom and the top of the ply as shown in Figure 1.

In equation (10), it is found that there are only five displacement variables in the displacement field of the proposed model RHSDTC. In order to assess the performance of the model RHSDTC, the generalized higher-order shear deformation theories with five displacement parameters [12] is chosen for comparison, which is given by

$$
\begin{aligned}
& u(x, y, z)=u_{0}(x, y)-z \frac{\partial w}{\partial x}+\Psi(z) u_{1}(x, y) \\
& v(x, y, z)=v_{0}(x, y)-z \frac{\partial w}{\partial y}+\Psi(z) v_{1}(x, y) \\
& w(x, y, z)=w(x, y)
\end{aligned}
$$


A Reddy-type higher-order shear deformation plate theory neglecting transverse normal strain (RHSDT) [11] can be given by setting function $\Psi(\mathrm{z})$ as

$$
\Psi(z)=z\left[1-\frac{4}{3}\left(\frac{z}{h}\right)^{2}\right]
$$

In addition, a displacement field of the sinusoidal shear deformation plate theory (SPT) [12] can be given by setting

$$
\Psi(z)=\frac{h}{\pi} \sin \left(\frac{\pi z}{h}\right)
$$

Karama et al. [25] proposed a higher-order shear deformation theory ESDT in which the function $\Psi(\mathrm{z})$ is set as

$$
\Psi(z)=z e^{-2(z / h)^{2}}
$$

A higher-order shear deformation theory FiSDT [23] has been proposed by setting function $\Psi(\mathrm{z})$ as

$$
\Psi(z)=\frac{7}{8} z-\frac{2 z^{3}}{h^{2}}+\frac{2 z^{5}}{h^{4}}
$$

A ninth-order theory HSDT-98 proposed by Matsunaga [32] will be employed to assess the performance of the generalized higher-order shear deformation models with five displacement parameters. The in-plane displacement field of the model HSDT-98 consists of 9th-order polynomial in the global thickness coordinate $z$ and the transverse deflection is represented by an 8th-order polynomial in $z$. Performance of the model HSDT-98 has been verified in reference [32], so results of HSDT-98 will be used as reference solutions to assess the performance of all other models. The ninth-order theory HSDT-98 is given by 


$$
\begin{aligned}
& u(x, y, z)=u_{0}(x, y)+\sum_{i=1}^{9} z^{i} u_{i} \\
& v(x, y, z)=v_{0}(x, y)+\sum_{i=1}^{9} z^{i} v_{i} \\
& w(x, y, z)=w(x, y)+\sum_{i=1}^{8} z^{i} w_{i}
\end{aligned}
$$

In equation (16), it is found that there are 29 variables in the displacement field of the mode HSDT-98.

\subsection{Constitutive equations}

For linear elasticity, the strain components of the model RHSDTC can be written as

$$
\begin{aligned}
\varepsilon_{x}= & \frac{\partial u_{0}}{\partial x}+\Phi_{1} \frac{\partial u_{1}}{\partial x}+\Phi_{2} \frac{\partial^{2} w_{0}}{\partial x^{2}}+\Phi_{3} \frac{\partial^{2} \bar{T}}{\partial x^{2}}+\Phi_{4} \frac{\partial^{2} \bar{C}}{\partial x^{2}} \\
\varepsilon_{y}= & \frac{\partial v_{0}}{\partial y}+\Psi_{1} \frac{\partial v_{1}}{\partial y}+\Psi_{2} \frac{\partial^{2} w_{0}}{\partial y^{2}}+\Psi_{3} \frac{\partial^{2} \bar{T}}{\partial y^{2}}+\Psi_{4} \frac{\partial^{2} \bar{C}}{\partial y^{2}} \\
\gamma_{x y}= & \frac{\partial u_{0}}{\partial y}+\Phi_{1} \frac{\partial u_{1}}{\partial y}+\frac{\partial v_{0}}{\partial x}+\Psi_{1} \frac{\partial v_{1}}{\partial x}+\left(\Phi_{2}+\Psi_{2}\right) \frac{\partial^{2} w_{0}}{\partial x \partial y} \\
& +\left(\Phi_{3}+\Psi_{3}\right) \frac{\partial^{2} \bar{T}}{\partial x \partial y}+\left(\Phi_{4}+\Psi_{4}\right) \frac{\partial^{2} \bar{C}}{\partial x \partial y} \\
\gamma_{x z}= & \frac{\partial \Phi_{1}}{\partial z} u_{1}+\left(1+\frac{\partial \Phi_{2}}{\partial z}\right) \frac{\partial w_{0}}{\partial x}+\frac{\partial \Phi_{3}}{\partial z} \frac{\partial \bar{T}}{\partial x}+\frac{\partial \Phi_{4}}{\partial z} \frac{\partial \bar{C}}{\partial x}+\frac{\partial w_{T}}{\partial x}+\frac{\partial w_{C}}{\partial x} \\
\gamma_{y z}= & \frac{\partial \Psi_{1}}{\partial z} v_{1}+\left(1+\frac{\partial \Psi_{2}}{\partial z}\right) \frac{\partial w_{0}}{\partial y}+\frac{\partial \Psi_{3}}{\partial z} \frac{\partial \bar{T}}{\partial y}+\frac{\partial \Psi_{3}}{\partial z} \frac{\partial \bar{C}}{\partial y}+\frac{\partial w_{T}}{\partial y}+\frac{\partial w_{C}}{\partial y}
\end{aligned}
$$

The temperature distribution $T$ and the moisture concentration $C$ through the thickness of plate are taken from reference [16]

$$
\begin{aligned}
& T(x, y, z)=\bar{T}_{0}(x, y)+(z / h) \bar{T}_{1}(x, y)+\left(f_{z} / h\right) \bar{T}_{2}(x, y) \\
& C(x, y, z)=\bar{C}_{0}(x, y)+(z / h) \bar{C}_{1}(x, y)+\left(f_{z} / h\right) \bar{C}_{2}(x, y)
\end{aligned}
$$

where $f_{z}=(h / \pi) \sin (\pi z / h)$.

The relationships between stresses and strains of the FGM plate subjected to hygrothermal load can be written as 


$$
\left\{\begin{array}{l}
\sigma_{x} \\
\sigma_{y} \\
\tau_{x y} \\
\tau_{x z} \\
\tau_{y z}
\end{array}\right\}=\left[\begin{array}{ccccc}
D_{11}(z) & D_{12}(z) & 0 & 0 & 0 \\
D_{21}(z) & D_{22}(z) & 0 & 0 & 0 \\
0 & 0 & D_{33}(z) & 0 & 0 \\
0 & 0 & 0 & D_{44}(z) & 0 \\
0 & 0 & 0 & 0 & D_{55}(z)
\end{array}\right]\left\{\begin{array}{c}
\bar{\varepsilon}_{x} \\
\bar{\varepsilon}_{y} \\
\bar{\gamma}_{x y} \\
\bar{\gamma}_{x z} \\
\bar{\gamma}_{y z}
\end{array}\right\}
$$

where $\quad \bar{\varepsilon}_{x}=\varepsilon_{x}-\alpha(z) \Delta T-\beta(z) \Delta C, \quad \bar{\varepsilon}_{y}=\varepsilon_{y}-\alpha(z) \Delta T-\beta(z) \Delta C, \quad \bar{\gamma}_{x y}=\gamma_{x y}$, $\bar{\gamma}_{x z}=\gamma_{x z}, \quad \bar{\gamma}_{y z}=\gamma_{y z} ; \quad D_{11}(z)=\frac{E(z)}{1-v^{2}}, \quad D_{12}(z)=D_{21}(z)=\frac{v E(z)}{1-v^{2}}, \quad D_{22}(z)=\frac{E(z)}{1-v^{2}}$ $D_{33}=D_{44}=D_{55}=\frac{E(z)}{2(1+v)} ; \alpha$ is the linear thermal expansion coefficients in the plate coordinates; $\beta$ is the moisture expansion coefficients; $\Delta T=T-\widehat{T}$ is the rise of temperature with respect to the reference temperature, where $\widehat{T}$ is the reference temperature; $\Delta C=C-\widehat{C}$ is the rise of moisture concentration with respect to the reference moisture concentration $\widehat{C}$.

\subsection{Equilibrium equations}

Applying the principle of minimum potential energy, the equilibrium equations for the model RHSDTC can be established. Rectangular Cartesian coordinates $(x, y, z)$ are used to describe the deformation of the FGM plate, in which $x \in\left[x_{0}, x_{a}\right]$ represents the plate longitudinal axis, $y \in\left[y_{0}, y_{b}\right]$ represents the plate width axis, and $z \in[-h / 2, h / 2]$ is the thickness coordinate. The principle of minimum potential energy can be expressed as

$$
\delta U-\delta W=0
$$

where the virtual strain energy $\delta U$ and virtual work $\delta W$ done by applied loads are respectively given by

$$
\begin{aligned}
& \delta U=\int_{A} \int_{-h / 2}^{h / 2}\left(\sigma_{x} \delta \varepsilon_{x}+\sigma_{y} \delta \varepsilon_{y}+\tau_{x y} \delta \gamma_{x y}+\tau_{x z} \delta \gamma_{x z}+\tau_{y z} \delta \gamma_{y z}\right) d z d x d y \\
& \delta W=\delta W_{p x}+\delta W_{p y}+\delta W_{q}+\delta W_{T x 0}+\delta W_{T x a}+\delta W_{T y 0}+\delta W_{T y b}
\end{aligned}
$$

where 


$$
\begin{aligned}
& \delta W_{p x}=-\iint_{A}\left[p_{x}^{b} \delta u\left(x, y, z_{1}\right)+p_{x}^{t} \delta u\left(x, y, z_{2}\right)\right] d x d y \\
& \delta W_{p y}=-\iint_{A}\left[p_{y}^{b} \delta v\left(x, y, z_{1}\right)+p_{y}^{t} \delta v\left(x, y, z_{2}\right)\right] d x d y \\
& \delta W_{q}=-\iint_{A}\left[q^{b} \delta w\left(x, y, z_{1}\right)+q^{t} \delta w\left(x, y, z_{2}\right)\right] d x d y \\
& \delta W_{T x 0}=\int_{-h / 2}^{h / 2}\left[T_{x 0} \delta u\left(x_{0}, y, z\right)+T_{x z 0} \delta w\left(x_{0}, y, z\right)\right] d z \\
& \delta W_{T y 0}=\int_{-h / 2}^{h / 2}\left[T_{y 0} \delta v\left(x, y_{0}, z\right)+T_{y z 0} \delta w\left(x, y_{0}, z\right)\right] d z \\
& \delta W_{T x a}=-\int_{-h / 2}^{h / 2}\left[T_{x a} \delta u\left(x_{a}, y, z\right)+T_{x z a} \delta w\left(x_{a}, y, z\right)\right] d z \\
& \delta W_{T y b}=-\int_{-h / 2}^{h / 2}\left[T_{y b} \delta v\left(x, y_{b}, z\right)+T_{y z b} \delta w\left(x, y_{b}, z\right)\right] d z
\end{aligned}
$$

where $p^{b}(x, y)$ and $p^{t}(x, y)$ are the distributed loads on the bottom and top surfaces of the plate; $q^{b}(x, y), q^{t}(x, y)$ are the transverse loads on the bottom and the top surfaces of the plate; $T_{x 0}, T_{x a}, T_{y 0}$ and $T_{y b}$ are the axial loads at the end cross-sections of the FGM plate; $T_{x z 0}, T_{x z a}, T_{y z 0}$ and $T_{y z b}$ are the transverse shear tractions at the end cross-sections of the plate.

By employing integration by parts and collecting the variational coefficients $\delta u_{0}$, $\delta u_{1}, \delta v_{0}, \delta v_{1}$ and $\delta w_{0}$, the equilibrium equations for the model RHSDTC are given by

$$
\begin{array}{ll}
\delta u_{0}: & N_{x, x}+N_{x y, y}+p_{x 0}=0 \\
\delta u_{1}: & M_{x \phi 1, x}+M_{x y \phi 1, y}-V_{\phi 1}+p_{x 1}=0 \\
\delta v_{0}: & N_{y, y}+N_{x y, x}+p_{y 0}=0 \\
\delta v_{1}: & M_{y \psi 1, y}+M_{x y \psi 1, x}-V_{\psi 1}+p_{y 1}=0 \\
\delta w_{0}: & M_{x \phi 2, x x}+M_{y \psi 2, y y}+M_{x y \phi 2, x y}+M_{x y \psi 2, x y} \\
& -V_{\phi 2, x}-V_{\psi 2, y}+p_{x 2, x}+p_{y 2, y}-q_{0}=0
\end{array}
$$

where

$$
\begin{aligned}
& {\left[N_{x}, M_{x \phi 1}, M_{x \phi 2}\right]=\int_{-h / 2}^{h / 2}\left[\sigma_{x}^{k}, \Phi_{1} \sigma_{x}^{k}, \Phi_{2} \sigma_{x}^{k}\right] d z ;} \\
& {\left[N_{y}, M_{y \phi 1}, M_{y \phi 2}\right]=\int_{-h / 2}^{h / 2}\left[\sigma_{y}^{k}, \Psi_{1} \sigma_{y}^{k}, \Psi_{2} \sigma_{y}^{k}\right] d z ;}
\end{aligned}
$$




$$
\begin{aligned}
& {\left[V_{\phi 1}, V_{\phi 2}\right]=\int_{-h / 2}^{h / 2}\left[\frac{\partial \Phi_{1}}{\partial z} \tau_{x z}^{k},\left(1+\frac{\partial \Phi_{2}}{\partial z}\right) \tau_{x z}^{k}\right] \mathrm{d} z} \\
& {\left[V_{\psi 1}, V_{\psi 2}\right]=\int_{-h / 2}^{h / 2}\left[\frac{\partial \Psi_{1}}{\partial z} \tau_{y z}^{k},\left(1+\frac{\partial \Psi_{2}}{\partial z}\right) \tau_{y z}^{k}\right] \mathrm{d} z ;} \\
& {\left[N_{x y}, M_{x y \phi 1}, M_{x y \phi 2}, M_{x y \psi 1}, M_{x y \psi 2}\right]=\int_{-h / 2}^{h / 2}\left[\tau_{x y}^{k}, \Phi_{1} \tau_{x y}^{k}, \Phi_{2} \tau_{x y}^{k}, \Psi_{1} \tau_{x y}^{k}, \Psi_{2} \tau_{x y}^{k}\right] d z ;} \\
& {\left[p_{x 0}, p_{x 1}, p_{x 2}\right]=\left[p_{x}^{b}+p_{x}^{t}, \Phi_{1}^{1} p_{x}^{b}+\Phi_{1}^{N} p_{x}^{t}, \Phi_{2}^{1} p_{x}^{b}+\Phi_{2}^{N} p_{x}^{t}\right]} \\
& {\left[p_{y 0}, p_{y 1}, p_{y 2}\right]=\left[p_{x}^{b}+p_{x}^{t}, \Psi_{1}^{1} p_{x}^{b}+\Psi_{1}^{N} p_{x}^{t}, \Psi_{2}^{1} p_{x}^{b}+\Psi_{2}^{N} p_{x}^{t}\right]} \\
& {\left[q_{0}\right]=\left[q^{b}+q^{t}\right] .}
\end{aligned}
$$

A set of consistent geometric (kinematic-variable) and kinetic (stress-resultant) boundary conditions [33] at the boundaries of the plates ( $x=x_{0}, x=x_{a}, y=y_{0}$ and $\left.y=y_{b}\right)$ can be expressed as

$$
\begin{array}{ll}
u_{0}\left(x_{\beta}, y\right)=\bar{u}_{0 \beta} & \text { or } N_{x}\left(x_{\beta}, y\right)=\bar{N}_{x \beta} \\
u_{1}\left(x_{\beta}, y\right)=\bar{u}_{1 \beta} & \text { or } M_{x \phi 1}\left(x_{\beta}, y\right)=\bar{M}_{x \phi 1 \beta} \\
v_{0}\left(x, y_{\lambda}\right)=\bar{v}_{0 \lambda} & \text { or } N_{y}\left(x, y_{\lambda}\right)=\bar{N}_{y \lambda} \\
v_{1}\left(x, y_{\beta}\right)=\bar{v}_{1 \lambda} & \text { or } M_{y \psi 1}\left(x, y_{\lambda}\right)=\bar{M}_{y \psi 1 \lambda} \\
w_{0, x}\left(x_{\beta}, y\right)=\bar{w}_{0, x \beta} & \text { or } M_{x \phi 2}\left(x_{\beta}, y\right)=\bar{M}_{x \phi 2 \beta} \\
w_{0, y}\left(x, y_{\lambda}\right)=\bar{w}_{0, y \lambda} & \text { or } M_{y \psi 2}\left(x, y_{\lambda}\right)=\bar{M}_{y \psi 2 \lambda} \\
w_{0}\left(x_{\beta}, y\right)=\bar{w}_{0 x \beta} & \text { or } \\
V_{\phi 2}\left(x_{\beta}, y\right)-M_{x \phi 2, x}\left(x_{\beta}, y\right)-M_{x y \phi 2, y}\left(x_{\beta}, y\right)-p_{x 2}\left(x_{\beta}, y\right)=\bar{V}_{x x \beta} \\
w_{0}\left(x, y_{\lambda}\right)=\bar{w}_{0 y \lambda} & \text { or } \\
V_{\psi 2}\left(x, y_{\lambda}\right)-M_{y \psi 2, y}\left(x, y y_{\lambda}\right)-M_{x y \psi 2, x}\left(x, y_{\lambda}\right)+p_{y 2}(x, y)=\bar{V}_{y x \lambda}
\end{array}
$$

where $\beta=x_{0}, x_{a} ; \lambda=y_{0}, y_{b}$;

$$
\begin{aligned}
& {\left[\bar{N}_{x \beta}, \bar{M}_{\phi 1 \beta}, \bar{M}_{\phi 2 \beta}\right]=\int_{-h / 2}^{h / 2}\left[T_{x \beta}, \Phi_{1} T_{x \beta}, \Phi_{2} T_{x \beta}\right] \mathrm{d} z ;} \\
& {\left[\bar{N}_{y \lambda}, \bar{M}_{\psi 1 \lambda}, \bar{M}_{\psi 2 \lambda}\right]=\int_{-h / 2}^{h / 2}\left[T_{y \lambda}, \Psi_{1} T_{y \lambda}, \Psi_{2} T_{y \lambda}\right] \mathrm{d} z}
\end{aligned}
$$




$$
\begin{aligned}
& {\left[\bar{V}_{x x \beta}\right]=\int_{-h / 2}^{h / 2}\left[T_{x z \beta}\right] \mathrm{d} z,(\beta=0, a) ;} \\
& {\left[\bar{V}_{y x \lambda}\right]=\int_{-h / 2}^{h / 2}\left[T_{y z \lambda}\right] \mathrm{d} z,(\lambda=0, b) ;}
\end{aligned}
$$

\subsection{Analytical solution}

Analytical solutions in terms of trigonometric functions for the FGM plates with simply-supported boundaries are considered. The external force, the temperature loads and moisture concentration loads are given by

$$
\begin{aligned}
& q=q_{0} \sin (\alpha x) \sin (\beta y) \\
& \bar{T}_{i}=T_{i} \sin (\alpha x) \sin (\beta y) \\
& \bar{C}_{i}=C_{i} \sin (\alpha x) \sin (\beta y)
\end{aligned}
$$

where $i=0,1,2$.

The simply-supported boundary conditions along the edges of the FGM plate are given as follows:

$$
\begin{aligned}
& \text { At edges } x=0 \text { and } x=a ; \\
& v_{0}=0, v_{1}=0, w_{0}=0 \\
& \text { At edges } y=0 \text { and } y=b ; \\
& u_{0}=0, u_{1}=0, w_{0}=0
\end{aligned}
$$

Following the Navier's procedure [31], analytical solution in terms of the displacement parameters satisfying the simply-supported boundary conditions are expressed as a double trigonometric series, such that

$$
\begin{aligned}
& u_{0}=\sum_{m=1}^{\infty} \sum_{n=1}^{\infty} u_{0 m n} \cos \alpha x \sin \beta y \\
& u_{1}=\sum_{m=1}^{\infty} \sum_{n=1}^{\infty} u_{1 m n} \cos \alpha x \sin \beta y \\
& v_{0}=\sum_{m=1}^{\infty} \sum_{n=1}^{\infty} v_{0 m n} \sin \alpha x \cos \beta y \\
& v_{1}=\sum_{m=1}^{\infty} \sum_{n=1}^{\infty} v_{1 m n} \sin \alpha x \cos \beta y \\
& w_{0}=\sum_{m=1}^{\infty} \sum_{n=1}^{\infty} w_{0 m n} \sin \alpha x \sin \beta y
\end{aligned}
$$


where $\alpha=m \pi / a ; \beta=n \pi / b ; a$ and $b$ denote respectively the length and the width of plates. Substituting equations (28) into equilibrium equations (23) and collecting coefficients, the following system equation can be obtained.

$$
\mathbf{K}_{5 \times 5} \mathbf{U}_{5 \times 1}=\mathbf{P}_{5 \times 1}
$$

where displacement parameters $\mathbf{U}=\left\{\begin{array}{lllll}u_{0} & u_{1} & v_{0} & v_{1} & w_{0}\end{array}\right\}^{T}$.

In equation (29), it is found that there are only five displacement variables in the model RHSDTC as transverse normal deformations $w_{T}$ and $w_{C}$ due to hygrothermal loads have been absorbed in the generalized force vector $\mathbf{P}_{5 \times 1}$.

\section{Results and discussion}

In this section, the static behavior of aluminum and alumina FGM plates subjected to hygro-thermo-mechanical loads is investigated by using the proposed model and other generalized higher-order models. The top surface of the FGM plate is ceramic (alumina) rich and the bottom surface is metal (aluminum) rich, which can be seen in Figure 1. Young's modulus, Poisson's ratio and coefficient of thermal expansion for aluminum are respectively $70 \mathrm{GPa}, 0.3,23\left(10^{-6} / \mathrm{)}\right)$, and for alumina are $380 \mathrm{GPa}, 0.3$ and $7.4\left(10^{-6} /{ }^{\circ} \mathrm{C}\right)$, respectively [18]. The effective Young's modulus $E(z)$, thermal expansion coefficient $\alpha(z)$ and moisture expansion coefficient $\beta(z)$ in the thickness direction of FGM plate can be computed using equation (1).

Example 1: Bending of a FGM square $(a=b)$ plate subjected to thermal loading $T(x, y)=T_{1}\left(\frac{2 z}{h}\right) \sin (\pi x / a) \sin (\pi y / b)$.

Transverse displacement is expressed in terms of the following dimensionless parameter.

$$
\hat{w}=\frac{w(a / 2, b / 2, z)}{h \alpha_{c} T_{1}}, \alpha_{c}=7.4 \times 10^{-6} /{ }^{\circ} \mathrm{C}
$$

Transverse displacements obtained using various models are presented in Table 1. It is remarked that results obtained from the models RHSDT, SPT, ESDT, FiSDT and 
HSDT-98 are recalculated in this paper. Numerical results showed that the transverse displacements obtained using the model HSDT-98 for the FGM plate subjected to thermal loading are in excellent agreement with those (HSDT-98*) obtained in reference [32] based on a similar analysis. As a result, the model HSDT-98 will be employed to assess the proposed model RHSDTC as well as other generalized higher-order models.

Example 2: The FGM plates subjected to hygro-thermo-mechanical loads $q=q_{0} \sin (\pi x / a) \sin (\pi y / b), \quad T(x, y, z)=\bar{T}_{0}(x, y)+(z / h) \bar{T}_{1}(x, y)+\left(f_{z} / h\right) \bar{T}_{2}(x, y) \quad$ and $C(x, y, z)=\bar{C}_{0}(x, y)+(z / h) \bar{C}_{1}(x, y)+\left(f_{z} / h\right) \bar{C}_{2}(x, y)$,

where $\left[\bar{T}_{0}(x, y), \bar{T}_{1}(x, y), \bar{T}_{2}(x, y)\right]=\left(T_{0}, T_{1}, T_{2}\right) \sin (\pi x / a) \sin (\pi y / b)$,

$$
\left[\bar{C}_{0}(x, y), \bar{C}_{1}(x, y), \bar{C}_{2}(x, y)\right]=\left(C_{0}, C_{1}, C_{2}\right) \sin (\pi x / a) \sin (\pi y / b)
$$

The dimensionless displacements and stresses are given by

$$
\begin{gathered}
\bar{u}=\frac{u(0, b / 2, z)}{h \alpha_{0} T_{0} a}, \bar{w}=\frac{w(a / 2, b / 2, z)}{h \alpha_{0} T_{0}}, \quad \bar{\sigma}_{x}=\frac{1}{E_{c} \alpha_{0} T_{0}} \sigma_{x}(a / 2, b / 2, z), \\
\bar{\tau}_{x y}=\frac{1}{E_{c} \alpha_{0} T_{0}} \tau_{x y}(0,0, z), \bar{\tau}_{x z}=\frac{1}{E_{c} \alpha_{0} T_{0}} \tau_{x z}(0, b / 2, z), E_{c}=380 \mathrm{GPa}, \alpha_{0}=10^{-6} /{ }^{\circ} \mathrm{C}
\end{gathered}
$$

This example illustrates the effects of temperature and moisture concentration on the displacements and the stresses of FGM plate. Figure 2 depicts the distributions of displacements and stresses through the thickness of the FGM plates subjected to hygro-thermo-mechanical loads $\left(q_{0}=100, T_{0}=T_{1}=100, T_{2}=0, C_{0}=C_{1}=3 \times 10^{-3}, C_{2}=0\right.$, $k=1)$. It is found that the results obtained from the model RHSDTC are in good agreement with those computed from the model HSDT-98 for thick $(a / h=2)$ and moderately thick $(a / h=4)$ FGM plates. However, the generalized higher-order theories RHSDT, SPT, ESPT and FiSDT are less accurate as transverse normal strain has been neglected in these models. For thin $(a / h=100)$ FGM plate, all models produce almost the same displacements and stresses. Numerical results show that hygrothermal response of thin FGM plates is insensitive to transverse normal strain, while 
transverse normal strain has significant impact on hygrothermal behaviors of thick FGM plates.

In Figure 3, the effect of volume fraction exponent $k$ on the displacements and stresses of the FGM plate subjected to hygro-thermo-mechanical load ( $q_{0}=100$, $\left.T_{0}=T_{1}=100, T_{2}=0, C_{0}=C_{1}=3 \times 10^{-3}, C_{2}=0, a / h=4\right)$ has been studied. The FGM plates are very sensitive to the change of volume fraction exponent $k$. The maximum absolute values of all results increase with the increase of the volume fraction exponent $k$. Numerical results also show that the distribution of in-plane displacements obtained from the models RHSDT, SPT, ESPT and FiSDT is linear, while in-plane displacements obtained from the model RHSDTC are not linear across the thickness of the FGM plate. In addition, the model RHSDTC produces the minimum in-plane displacements near the middle surface of the FGM plate, while the models RHSDT, SPT, ESPT and FiSDT yield the minimum in-plane displacements at the bottom surface of the plate. It ought to be indicated that transverse shear stresses are obtained by integrating three-dimensional equilibrium equation through the thickness of plates. However, transverse shear stresses obtained from the models RHSDT, SPT, ESPT and FiSDT deviate substantially from those of HSDT-98 as shown in Figure 3. In addition, results obtained from the models RHSDT, SPT, ESPT and FiSDT are closer to those computed from the models RHSDTC and HSDT-98 with an increase of volume fraction exponent $k$.

Figure 4 depicts the effects of loading on the displacements and the stresses of the thick FGM plates $(a / h=2)$. For hygrothermal expansion of FGM plate $\left(q_{0}=100\right.$, $\left.T_{0}=100, T_{1}=T_{2}=0, C_{0}=3 \times 10^{-3}, C_{1}=C_{2}=0\right)$, the models RHSDT and FiSDT are pretty bad in producing reliable displacements and stresses. In terms of the models RHSDT and FiSDT, the minimum in-plane stresses $\bar{\sigma}_{x}$ occur at the bottom surface of plate. In fact, the minimum in-plane stresses $\bar{\sigma}_{x}$ obtained from the models RHSDTC and HSDT098 appear close to the middle surface. In addition the models RHSDTC and HSDT-98 produce the minimum in-plane displacements near the middle surface of the FGM plate, while the models RHSDT and FiSDT yield the minimum in-plane 
displacements at the top surface of the plate. Furthermore, transverse shear stresses obtained from the models RHSDT and FiSDT are close to zero, which completely differs from those computed from the models RHSDTC and HSDT-98. With increase of hygrothermal loads, in-plane displacement $\bar{u}$ and in-plane shear stress $\bar{\tau}_{x y}$ at the top surface of plate rapidly increase, whereas in-plane displacement and in-plane shear stress at the bottom surface of the plate rapidly decrease. However, transverse shear stresses are insensitive to hygrothermal loads. Figure 5 shows the effects of the aspect ratio $a / b$ on the displacement and the stresses of the FGM plate. The in-plane displacement $\bar{u}$ and the in-plane shear stress $\bar{\tau}_{x y}$ gradually decrease as the aspect ratio $a / b$ increases. However, the absolute values in-plane stresses $\bar{\sigma}_{x}$ and transverse shear stresses $\bar{\tau}_{x z}$ increase with the increase of the aspect ratio $a / b$.

\section{Conclusion}

A analytical model in terms of five displacement parameters has been developed for hygro-thermo-mechanical analysis of FGM plates. Compared to other generalized higher-order theories with five displacement parameters, the model RHSDTC takes into account the transverse normal strain without adding any extra degrees of freedom. Based on the proposed model, the equations of equilibrium have been developed by using the principle of minimum total potential energy. Analytical solutions for the simply-supported FGM plates subjected to hygro-thermo-mechanical loads are presented by applying the Navier procedure. Relative to the other models of the same number of displacement parameters, numerical results show that the model RHSDTC agrees particularly well with the analytical solution of the model HSDT-98 with 29 displacement variables. The influence of the temperature, moisture concentration, volume fraction, the thickness-to-side ratio and the aspect ratio on displacements and the stresses is very significant as shown in the case of the simply-supported FGM plates. More importantly, it is found that distributions of displacements and stresses through the thickness obtained from the proposed model RHSDTC generally differ 
from those obtained from other generalized higher-order models of thick FGM plates. From the computed results, it is found that the proposed model is very promising to offer a simple and effective solution to FGM plate structures subjected to hygro-thermo-mechanical loads.

\section{Acknowledgement}

The work described in this paper has been supported by the Seed Funding Programme for Basic Research for the project "Modelling and analysis of curved composite laminated shell structures" (201410159028) of the University of Hong Kong and the National Natural Sciences Foundation of China [No. 11272217, 11402152].

\section{References}

[1] Zenkour AM, Exact solution of thermal stress problem of an inhomogeneous hygrothermal piezoelectric hollow cylinder. Applied Mathematical Modelling, 2014; 38:6133-6143.

[2] Kar VR, Panda SK, Thermoelastic analysis of functionally graded doubly curved shell panels using nonlinear finite element method. Compos Struct, 2015; 129:202-212.

[3] Taibi FZ, Benyoucef S, Tounsi A, Bouiadjra RB, Bedia EAA, Mahmoud SR, A simple shear deformation theory for thermo-mechanical behaviour of functionally graded sandwich plates on elastic foundations. Journal of Sandwich Structures and Materials, 2015; 17:99-129.

[4] Sundararajan N, Prakash T, Ganapathi M, Nonlinear free flexural vibrations of functionally graded rectangular and skew plates under thermal environment. Finite Elem Anal Des, 2005; 42:152-168.

[5] Nguyen-Xuan H, Tran LV, Nguyen-Thoi T, Vu-Do HC, Analysis of functionally graded plates using an edge-based smoothed finite element method. Compos Struct, 2011; 93:3019-3039. 
[6] Singha MK, Prakash T, Ganapathi, Finite element analysis of functionally graded plates under transverse load. Finite Elem Anal Des, 2011; 47:453-460.

[7] Nguyen-Xuan H, Tran LV, Thai CH, Nguyen-Thoi T, Analysis of functionally graded plates by an efficient finite element method with node-based strain smoothing. Thin-Walled Structures, 2012; 54:1-18.

[8] Matsunaga H, Assessment of a global higher-order deformation theory for laminated composite and sandwich plates. Compos Struct, 2002; 56:279-291.

[9] Ferreira AJM, Castro LMS, Bertoluzza S, A higher order collocation method for the static and vibration analysis of composite plates using a first-order theory. Compos Struct, 2003; 34:627-636.

[10] Reddy JN, Analysis of functionally graded plates. Int J Numer Methods Eng, $2000 ; 47: 663-684$.

[11] Reddy JN, A simple higher-order theory for laminated composite plates. J Appl Mech, 1984; 51:745-752

[12] Zenkour AM, Generalized shear deformation theory for bending analysis of functionally graded plates. Applied Mathematical Modelling, 2006; 30:67-84.

[13] Touratier M, An efficient standard plate theory. Int J Eng Sci, 1991; 29:901-916.

[14] Zenkour AM, Alghamdi NA, Thermoelastic bending analysis of functionally graded sandwich plates. J Mater Sci, 2008; 43:2574-2589.

[15] Zenkour AM, The refined sinusoidal theory for FGM plates on elastic foundations. Int J Mech Sci, 2009; 51:869-880.

[16] Zenkour AM, Hygro-thermo-mechanical effects on FGM plates resting on elastic foundations. Compos Struct, 2010; 93:234-238.

[17] Zenkour AM, Alghamdi NA, Bending analysis of functionally graded sandwich plates under the effect of mechanical and thermal loads. Mechanics of Advanced Materials and Structures, 2010; 17:419-432. 
[18] Zenkour AM, Alghamdi NA, Thermomechanical bending response of functionally graded nonsymmetric sandwich plates. Journal of Sandwich Structures and Materials, 2010; 12:7-46.

[19] Zenkour AM, Allam MNM, Sobhy M, Bending analysis of FG viscoelastic sandwich beams with elastic cores resting on Pasternak's elastic foundation. Acta Mech, 2010; 212:233-252.

[20] Zenkour AM, Sobhy M, Elastic foundation analysis of uniformly loaded functionally graded viscoelastic sandwich plates. Journal of Mechanics, 2012; 28:439-452.

[21] Zenkour AM, Sobhy M, Dynamic bending response of thermoelastic functionally graded plates resting on elastic foundation. Aerospace Science and Technology, $2013 ; 29: 7-17$.

[22] Sobhy M, Buckling and free vibration of exponentially graded sandwich plates resting on elastic foundations under various boundary conditions. Compos Struct, 2013; 99:76-87.

[23] Nguyen-Xuan H, Thai HC, Nguyen-Thoi T, Isogeometric finite element analysis of composite sandwich plates using a higher order shear deformation theory. Composite Part B, 2013; 55:558-574.

[24] Arya H, Shimpi RP, Naik NK, A zig-zag model for laminated composite beams. Compos Struct, 2002; 56:21-24.

[25] Karama M, Afaq KS, Mistou S, Mechanical behavior of laminated composite beam by new multi-layered laminated composite structures model with transverse shear stress continuity. Int J Solids Struct, 2003; 40:1525-1546.

[26] Thai CH, Kulasegaram S, Tran LV, Nguyen-Xuan H, Generalized shear deformation theory for functionally graded isotropic and sandwich plates based on isogeometric approach. Comput Struct, 2014; 141:94-112.

[27] Ali JSM, Bhaskar K, Varadan TK, A new theory for accurate thermal/mechanical 
flexural analysis of symmetric laminated plates. Compos Struct, 1999; 45:227-232.

[28] Cho M, Oh J, Higher order zig-zag theory for fully coupled thermo-electricmechanical smart composite plates. Int J Solids Struct, 2004; 41:1331-1356.

[29] Carrera E, Transverse normal strain effects on thermal stress analysis of homogeneous and layered plates. AIAA J, 2005; 43:2232-2242.

[30] Chen WJ, Wu Z, A selective review on recent development of displacement-based laminated plate theories. Recent Patents on Mechanical Engineering, 2008; 1:29-44.

[31] Kant T, Swaminathan K. Analytical solutions for the static analysis of laminated composite and sandwich plates based on a higher order refined theory. Compos Struct, 2002; 56:329-344.

[32] Matsunaga $\mathrm{H}$, Stress analysis of functionally graded plates subjected to thermal and mechanical loading. Compos Struct, 2009; 87:344-357.

[33] Gherlone M, Tessler A, Di Sciuva M, $\mathrm{C}^{0}$ beam elements based on the Refined Zigzag Theory for multilayered composite and sandwich laminates. Compos Struct, 2011; 93:2882-2894. 


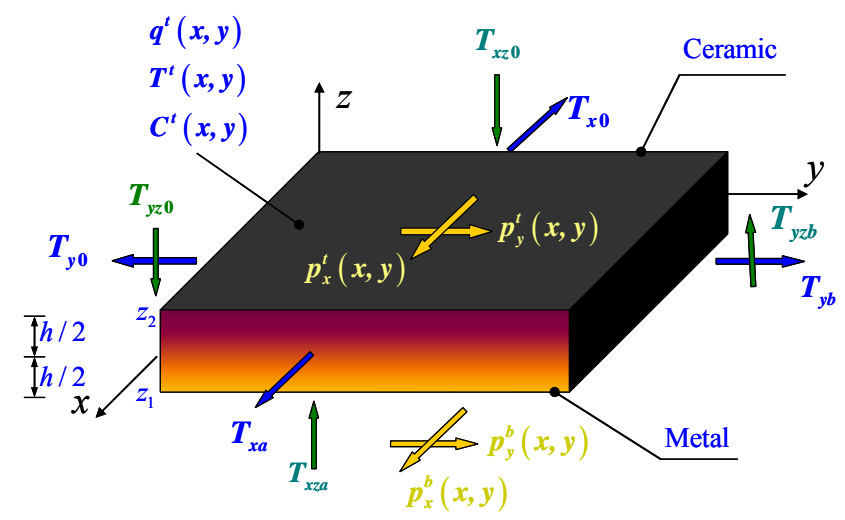

Fig. 1 A FGM plate subjected to various loads 

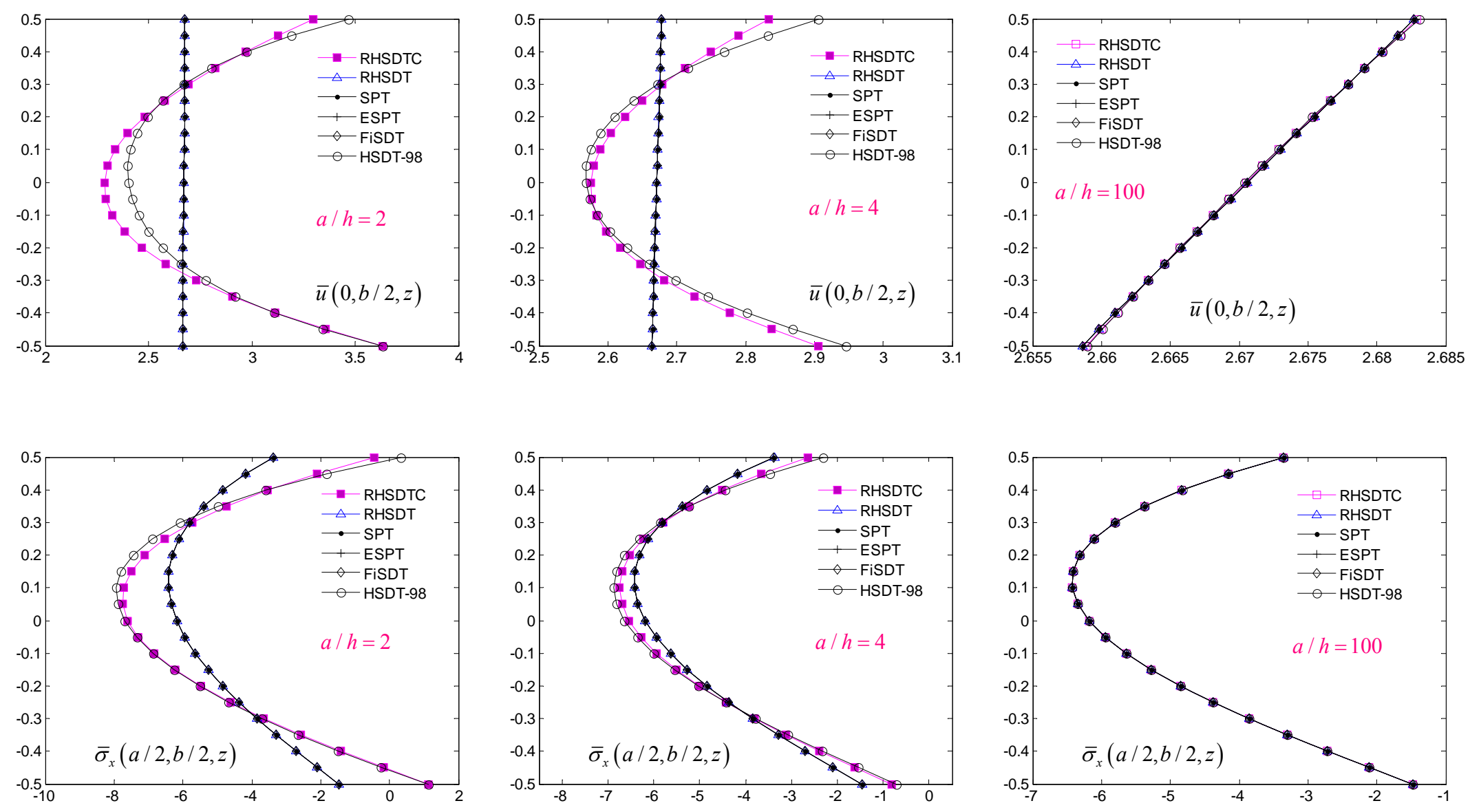

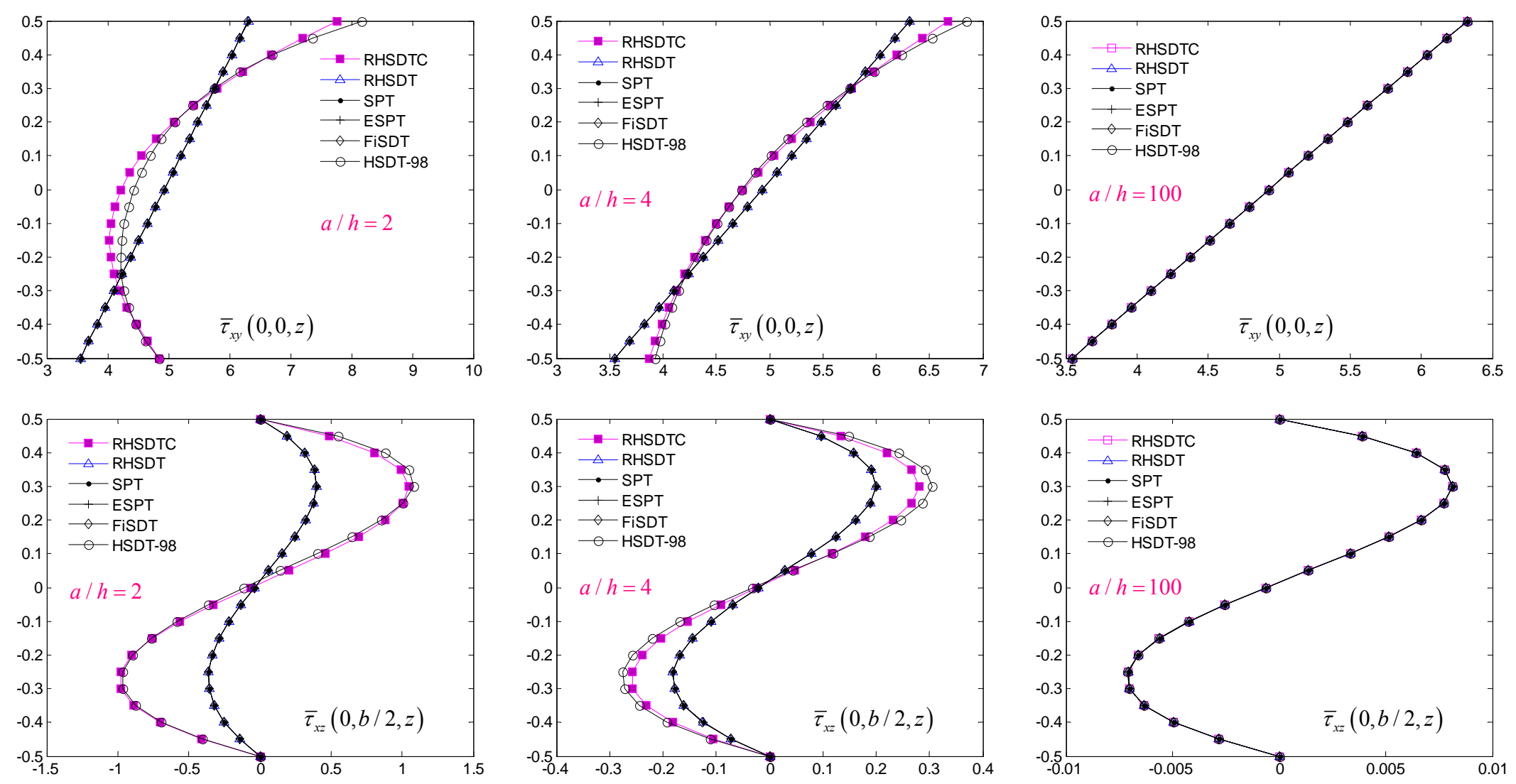

Fig. 2 Comparison of displacement and stresses through thickness of the FGM plate subjected to thermomechanical loading $\left(k=1, q_{0}=100\right.$,

$$
\left.T_{0}=T_{1}=100, T_{2}=0, C_{0}=C_{1}=3 \times 10^{-3}, C_{2}=0\right)
$$



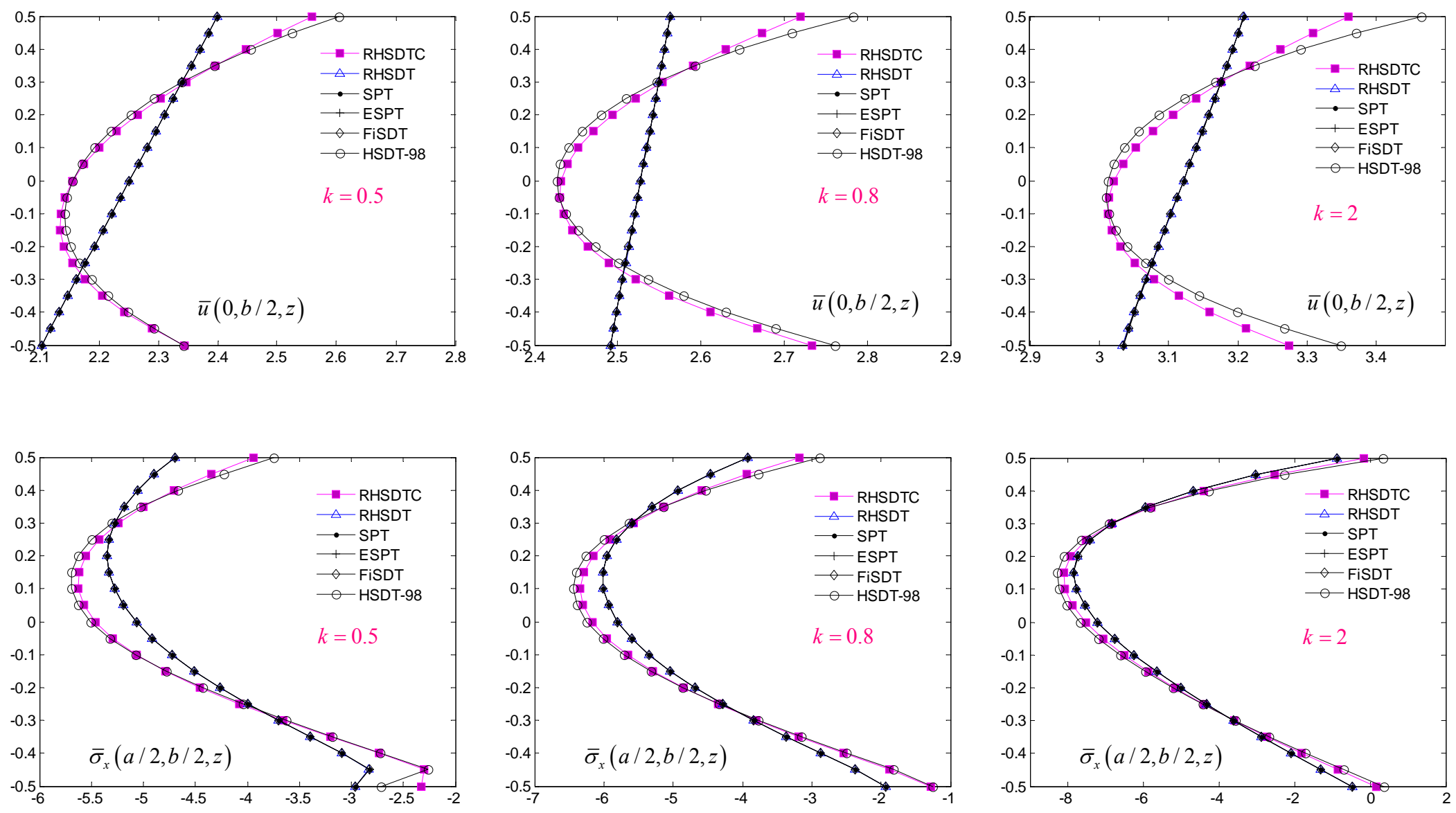

25 

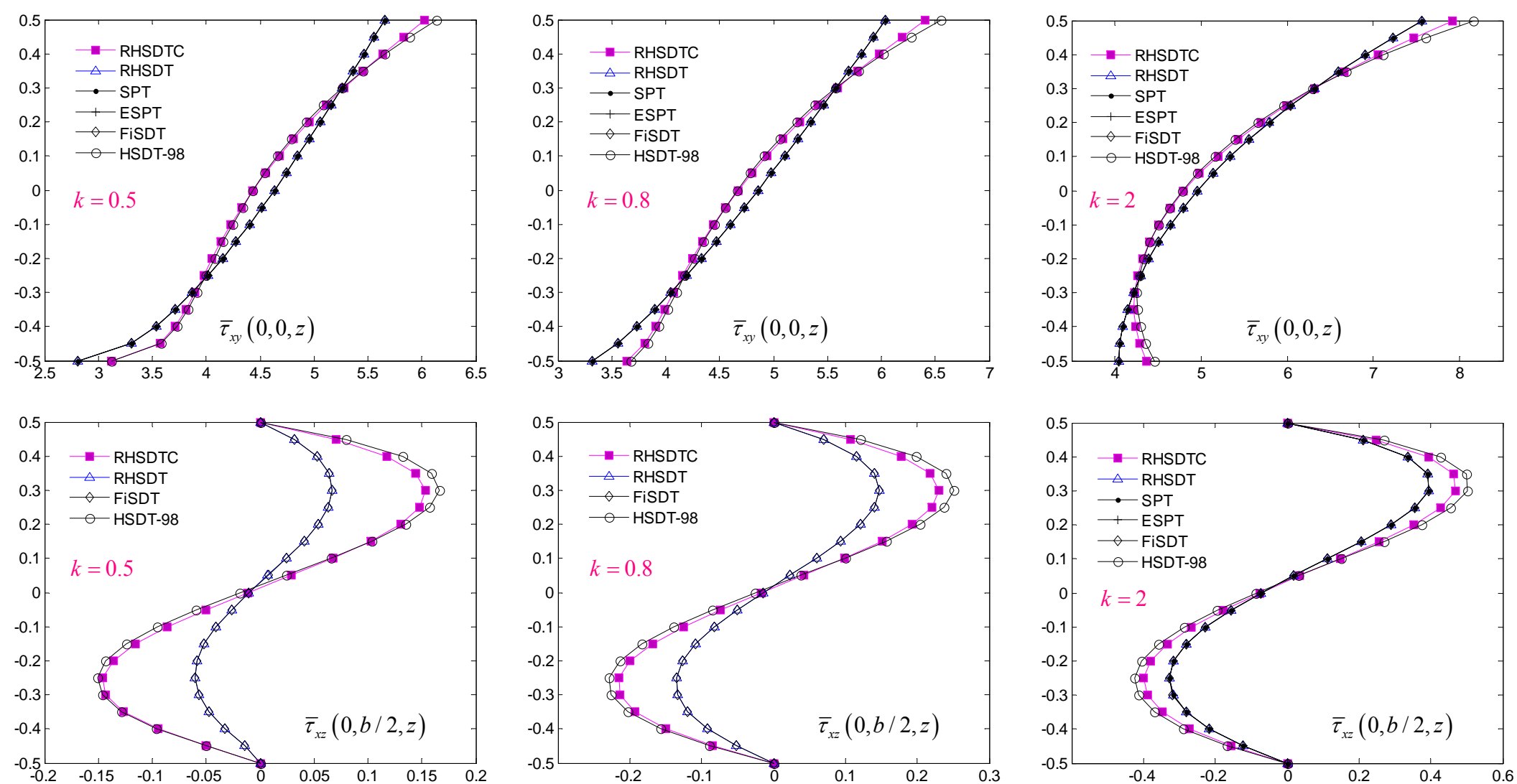

Fig. 3 Comparison of displacement and stresses through thickness of the FGM plate subjected to thermomechanical loading $\left(a / h=4, q_{0}=100\right.$,

$$
T_{0}=T_{1}=100, T_{2}=0, C_{0}=C_{1}=3 \times 10^{-3}, C_{2}=0 \text { ) }
$$



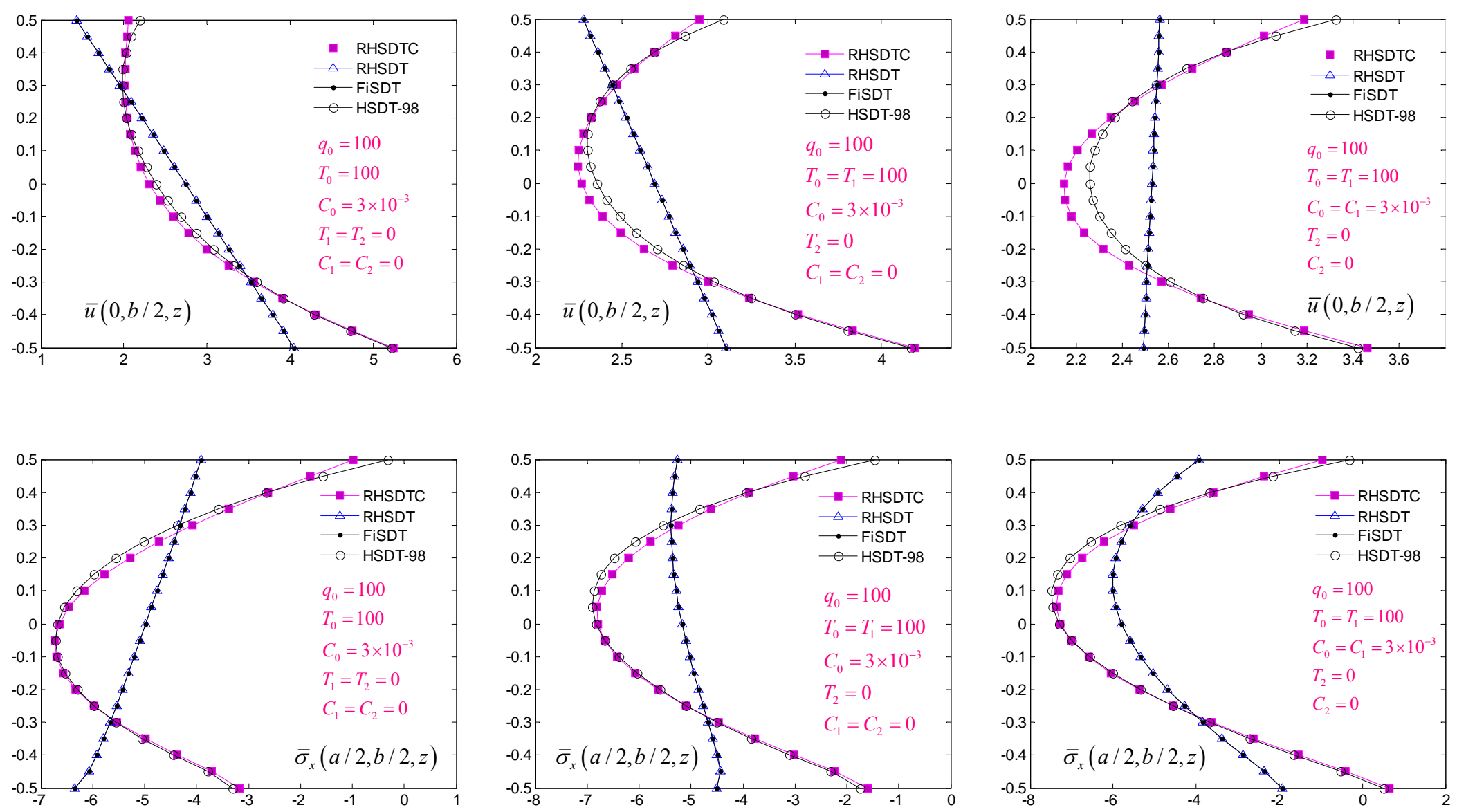

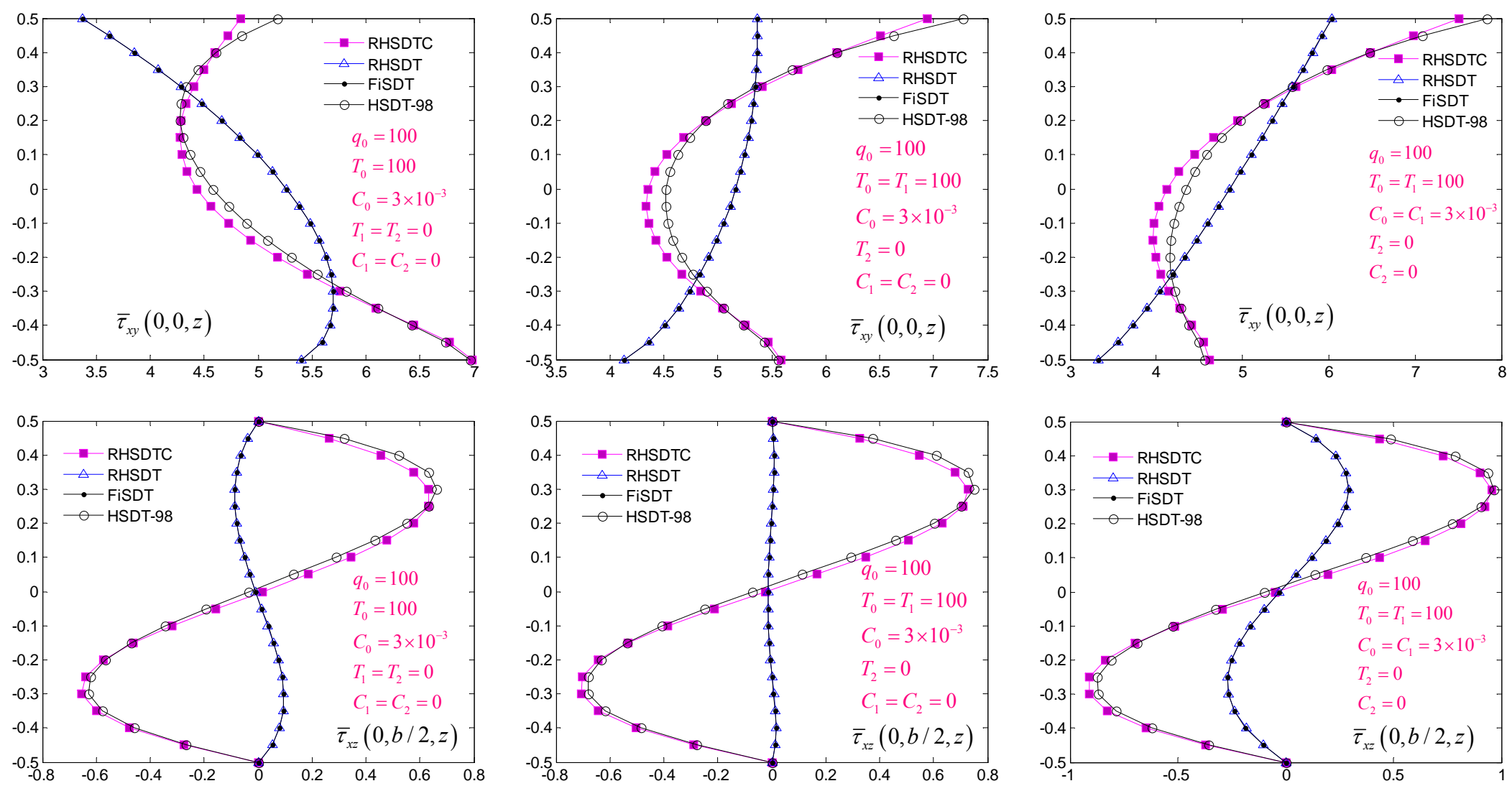

Fig. 4 Comparison of displacement and stresses through thickness of the FGM plate subjected to various loadings $(a / h=2, k=0.8)$ 

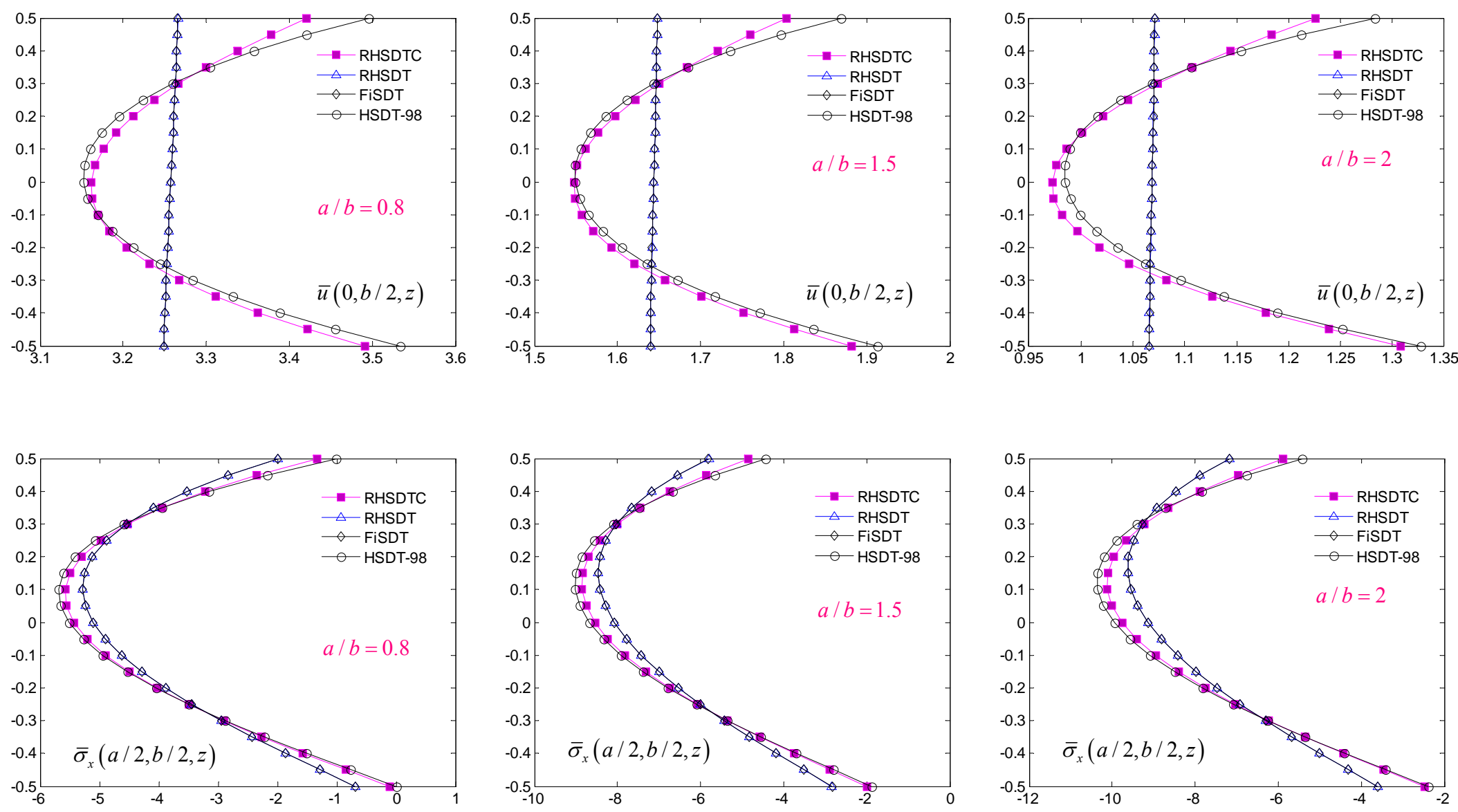

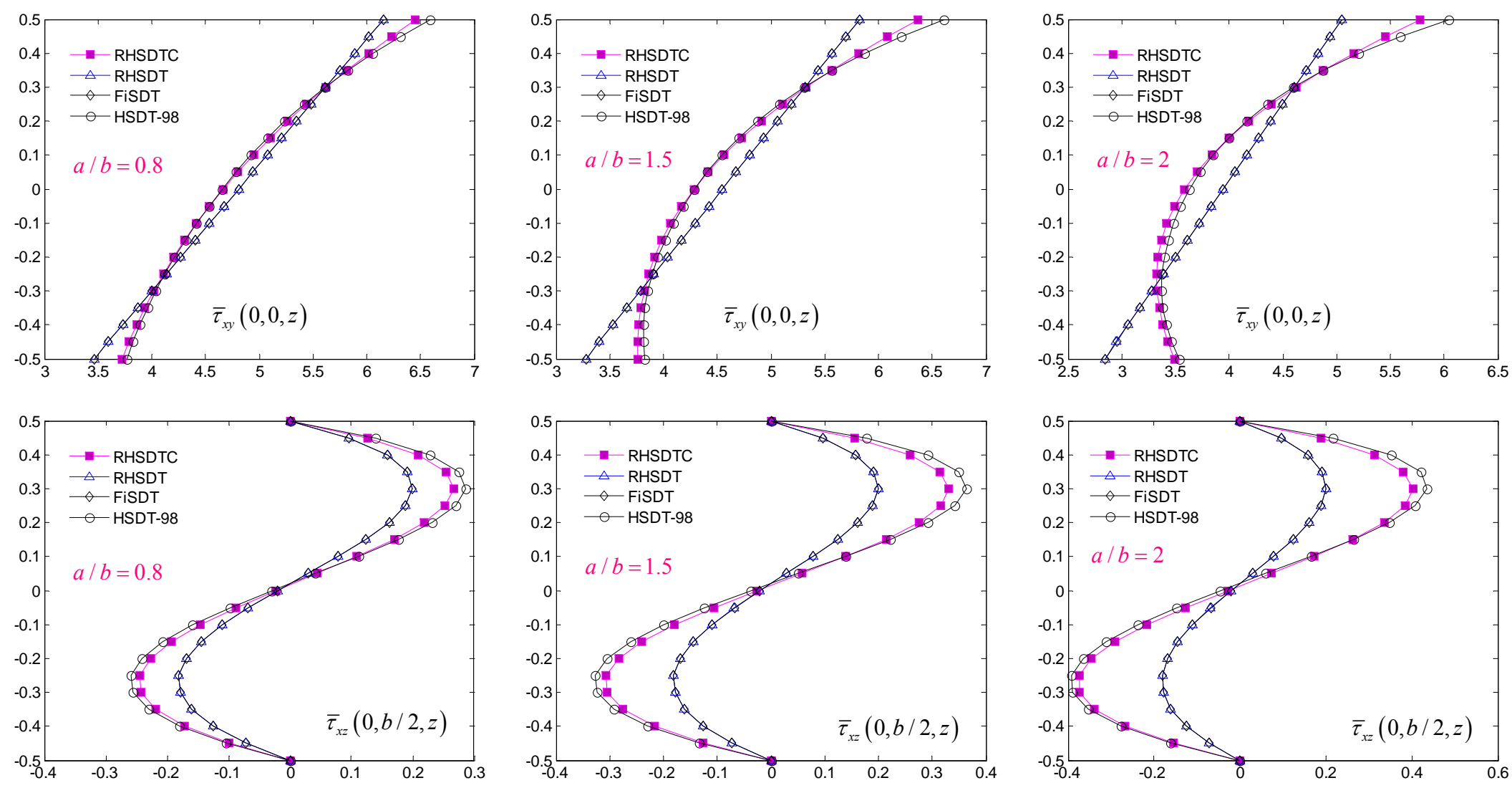

Fig. 5 Comparison of displacement and stresses through thickness of the FGM plate subjected to various loadings $\left(a / h=4, k=1, q_{0}=100\right.$,

$$
\left.T_{0}=T_{1}=100, T_{2}=0, C_{0}=C_{1}=3 \times 10^{-3}\right)
$$


Table 1 Transverse displacement $\hat{w}$ of the FGM plate subjected to thermal loading $\left(q_{0}=0, T_{1}=100, T_{0}=T_{2}=0, C_{0}=C_{1}=C_{2}=0\right)$

\begin{tabular}{|l|l|l|l|l|}
\hline$a / h$ & Models & $k=0$ & $k=0.5$ & $k=1$ \\
\hline 5 & RHSDTC & 3.2929 & 5.3949 & 5.9910 \\
\hline & ESDT & 3.2929 & 5.3943 & 5.9877 \\
\hline & FiSDT & 3.2929 & 5.3938 & 5.9856 \\
\hline & HSDT-98 & 3.2270 & 5.2855 & 5.8415 \\
\hline 10 & HSDT-98* [32] & 3.227 & 5.286 & 5.842 \\
\hline & RHSDTC & 13.1717 & 21.5896 & 24.0103 \\
\hline & ESDT & 13.1717 & 21.5890 & 24.0070 \\
\hline & FiSDT & 13.1717 & 21.5885 & 24.0048 \\
\hline & HSDT-98 & 13.1065 & 21.4803 & 23.8602 \\
\hline & HSDT-98* $[32]$ & 13.11 & 21.48 & 23.86 \\
\hline
\end{tabular}

